\title{
Quantitative Real-Time RT-PCR Using Hybridization Probes and Imported Standard Curves for Cytokine Gene Expression Analysis
}

BioTechniques 33:1078-1089 (November 2002)

\section{Benedikte Sabina Kühne and Patrick Oschmann University of Gießen, Gießen, Germany}

\section{INTRODUCTION}

Cytokines are implicated in many cellular processes. Variations in the amount or pattern of expression of the genes coding for these molecules contribute to immune-mediated disorders such as multiple sclerosis. Therefore, the detection and quantification of cytokine mRNA levels are of fundamental importance for understanding diverse pathological states and for monitoring therapeutic effects (7). Since real-time or kinetic PCR was first described in 1993 (6), it has been increasingly used to quantify copy numbers of mRNAs for gene expression analysis (quantitative RT-PCR), especially in clinical applications. The principle of the methodology permits the direct measurement of PCR product accumulation during the log-linear phase of the reaction, combining amplification, detection, and quantification in a single step (2). This allows more accurate measurement compared to the traditional endpoint analysis.

The first step in an RT-PCR assay is the reverse transcription of the RNA template into cDNA. In general, there are two strategies that can be followed. Using a one-step RT-PCR reduces handling and time but requires separate reverse transcription reactions for every parameter studied. Therefore, when screening many different parameters, a two-step RT-PCR is advantageous. For amplification of cDNAs, the LightCycler $^{\mathrm{TM}}$ (Roche Applied Science, Mannheim, Germany) (11), one of the commercially available real-time PCR systems, was used. This is a rapid thermal cycler with online fluorescence detection, which performs the PCR in glass capillaries contained within a rotor-like carousel. The capillaries are heated and cooled in an airstream. For sequence-specific detection, the signal to be analyzed can be generated using hybridization probes (10), which are pairs of oligonucleotides labeled with donor or acceptor fluorophores. These need to bind in close proximity to ensure an efficient fluorescence resonance energy transfer (FRET) (3).

There are in general two methods for quantification of specific cDNA targets (5). The absolute quantification method determines an absolute value for the target concentration (i.e., copy number) by using an external standard with known concentrations. The relative quantification method describes the change in expression of the target gene in relation to a reference such as an endogenous housekeeping gene (1) or an unstimulated control (9).

By using a combination of both methods, we have developed quantitative two-step RT-PCR assays for five parameters. For absolute quantification, external standard curves were constructed using the appropriate homologous DNA plasmid for each parameter. The absolute values of the measured cytokines were set in relation to the endogenous gene expression of human porphobilinogen deaminase (PBGD), a housekeeping gene not known to have pseudogenes (4). The level of PBGD was used for normalization of the samples, thereby acting as an internal control to compensate for variations in the sample volume, target recovery during nucleic acid extraction, and the presence of inhibitors. A new feature of the LightCycler software version 3.5 was used for absolute quantification. This 
verse transcriptase, and quick-chilling to $4^{\circ} \mathrm{C}$. The cDNA was stored at $-20^{\circ} \mathrm{C}$.

\section{Oligonucleotides}

Primers and hybridization probes (Table 1) were designed and delivered by TIB Molbiol (Berlin, Germany). The synthesis of intron-spanning primers prevented amplification of genomic DNA. The hybridization probes consist of two different oligonucleotides that bind to specific internal sequences of the amplified fragments during the annealing phase of PCR cycles in such order that the donor-probe ( $3^{\prime}$-fluorescein) and the acceptor-probe ( $5^{\prime}$-LC Red 640) hybridize head-to-tail in close proximity (1-5 nucleotides). This is necessary for an efficient FRET process.

\section{Plasmid Standards}

The plasmids served as external homologous DNA standards of known copy numbers to produce standard curves for each specific cytokine target and for the housekeeping gene PBGD, which serves as an internal control. All plasmid standards were cloned by GenExpress (Berlin, Germany). For more information, see Table 1.

JM 109 high-efficiency competent cells (Promega) were used for the transformation of the plasmids according to the manufacturer's protocol. The plasmids were isolated with the HiSpeed ${ }^{\mathrm{TM}}$ Plasmid Purification Kit (Qiagen), linearized by HindIII (Roche Applied Science), and purified with the QIAquick $^{\mathrm{TM}}$ PCR Purification Kit (Qiagen) following the manufacturer's instructions. The plasmids were checked by agarose gel electrophoresis and PCR (SYBR $^{\circledR}$ Green I melting curve analysis) for the proper signal. Plasmid DNA quality and concentration were evaluated by absorbance spectroscopy at 260 , 280, and $320 \mathrm{~nm}$ using an Ultraspec ${ }^{\mathrm{TM}}$ 2000 spectrophotometer (Amersham Biosciences, Cambridge, UK).

The copy number of each standard was calculated by using the Avogadro constant and the size of the plasmid used.

\section{Construction of the External Plasmid Standard Curves}

For the quantification of the different cytokine targets and of PBGD, the appropriate homologous plasmid standard was used to make sure that the cDNA and the standard were amplified with at least similar efficiency. To generate the standard curves, the plasmids were serially diluted in 1:10 steps with MS2 RNA (final concentration, $10 \mathrm{ng} / \mu \mathrm{L}$ ) (Roche Applied Science) in siliconized tubes. The MS2 RNA served as the background nucleic acid to prevent binding of standard DNA to the sides of the tubes. The log range of the different standard curves was dependent on the cytokine target. Usually a range from $10^{5}$ to $10^{0}$ copies was used. The dilutions of $10^{3}$ copies were aliquoted in siliconized tubes and stored at $-20^{\circ} \mathrm{C}$.

Only these aliquots were used as the standard triplicate (standard duplicate and unknown) in the different PCR runs for the quantification of the target cDNAs (see Importing External Standard Curves to Different PCR Runs section). The software version 3.5 of the LightCycler system offers the opportunity to generate an external standard curve for each parameter, which can be imported to different PCR runs. To generate a reliable standard curve for each parameter, they were produced with 5-fold measurements for each standard dilution point over the whole standard curve range. To check for reproducibility, the standard curves were repeated on different days. The standard curves should have a mean squared error $\leq 0.05$ and a slope near -3.3 , and therefore an efficiency near $2.0\left(\mathrm{E}=10^{-1 / \text { Slope }}\right)$.

\section{Quantitative Real-Time PCR}

The LightCycler system was used for amplification and data collection (software version 3.5). All reactions were performed with the LightCycler-FastStart DNA Master Hybridization Probes (Roche Applied Science). This is a ready-to-use, "hot-start" reaction mixture for PCR containing FastStart Taq DNA polymerase, which is inactive at room temperature because of heat-labile blocking groups on some of the amino acid residues of the enzyme. Therefore, it minimizes nonspecific amplification products. Reactions were carried out in a total volume of $20 \mu \mathrm{L} /$ capillary. Each reaction master mixture contained 4
$\mathrm{mM} \mathrm{MgCl}_{2}, 0.5 \mu \mathrm{M}$ each primer, 0.15 $\mu \mathrm{M}$ each hybridization probe, $1 \times$ LightCycler-FastStart DNA Master Hybridization probes, and water. It was mixed at $4{ }^{\circ} \mathrm{C}$ and distributed into the prechilled capillaries. For quantification of the cytokines, $2 \mu \mathrm{L}$ plasmid standard dilution $\left(10^{3}\right.$ in triplicate; see Importing External Standard Curves to Different PCR Runs section) or $2 \mu \mathrm{L}$ cDNA were added. In every run, a negative control (water) was included to check for cross contamination.

\section{Amplification Protocol}

A pre-incubation step of $10 \mathrm{~min}$ at $95^{\circ} \mathrm{C}$ to activate the Taq DNA polymerase was followed by 45 amplification cycles (IFN- $\beta, 50$ cycles) consisting of $10 \mathrm{~s}$ at $95^{\circ} \mathrm{C}, 10 \mathrm{~s}$ at $60^{\circ} \mathrm{C}$, and $15 \mathrm{~s}$ at $72^{\circ} \mathrm{C}$ (temperature ramp always $20^{\circ} \mathrm{C} / \mathrm{s}$ ). The fluorescence measurements were made at the end of the annealing phase at $60^{\circ} \mathrm{C}$. The PCR raw data were analyzed with the LightCycler software version 3.5 . The ratio of the signals measured in channel 2/channel 1 was used to calculate the crossing point $(\mathrm{Cp}$ ) values (Second Derivative Maximum method; proportional baseline adjustment). The $\mathrm{Cp}$ value represents the cycle number in which the fluorescence increases for the first time above a threshold value.

\section{Optimization Strategy}

Two RNA isolation kits (from Qiagen and Roche Applied Science) were compared for the quality and yield of extracted RNA. The reverse transcription was checked for linearity by variation of the amount of RNA fit into the reactions. Also, different reverse transcriptases (from Invitrogen, Roche Applied Science, and Qiagen) were compared.

PCR conditions were optimized for $\mathrm{MgCl}_{2}$ concentration (1-5 mM) with different primer combinations under use of dilutions $\left(10^{5}, 10^{4}\right.$, and $\left.10^{3}\right)$ of plasmid standard and cDNA $(1: 1$ and $1: 10)$. Products were analyzed with SYBR Green I staining and melting curve analysis to identify specific PCR products and primer dimers by differences in $\mathrm{T}_{\mathrm{m}}$. For adaptation to the hybridization probe format, an additional $\mathrm{MgCl}_{2}$ titration (1-5 mM) in the pres- 
ence of hybridization probes is necessary. The reactions were also checked with agarose gel electrophoresis to correlate product length with melting peaks. To check for possible DNA contamination (incomplete DNase digestion), total RNA was amplified in PCR for 45 cycles, using the chosen primerprobe pair without previous reverse transcription reaction (RT-minus control). Other optimization parameters included the variation of primer and probe concentrations, annealing temperature, and a control of possible inhibitors (EDTA, heparin, and porphyrins from blood samples). To compare PCR amplification efficiency of cDNA from a multiple sclerosis patient to that of the homologous plasmid standard, a dilution series of cDNA (serial 1:10 in MS2 RNA) was PCR-amplified (every point was measured in quintuple) with every parameter. The copy numbers of the different parameters were quantified with the appropriate imported plasmid standard curve.

\section{RESULTS AND DISCUSSION}

Cytokines are regulatory proteins that play an important role in many cellular processes such as proliferation, regulation of cell differentiation, and cell-cell communication. The analysis of cytokine profiles is important to elucidate the pathological pathways involved in immune-mediated disorders such as multiple sclerosis and for monitoring the effects of an immunomodulatory therapy. Quantitative real-time RTPCR is an accurate and sensitive method to quantify the mRNA expression of cytokines and is becoming more widely used compared to the alternative of endpoint measurement of conventional RTPCR. A recent study showed that realtime RT-PCR for IL-16 mRNA quantification gave similar results compared to quantitative competitive RTPCR (1), the previously most widely accepted method of quantification. Since real-time RT-PCR using the LightCycler is significantly less time consuming, the quantitative RT-PCR method we describe is an advantageous technique, especially for clinical applications.

A real-time, online, two-step quantitative RT-PCR method was established for gene expression analysis of cytokines and their receptors from blood leucocytes of multiple sclerosis patients. The LightCycler system was used to quantify the different targets on the basis of imported external plasmid standard curves using the hybridization probe format.

\section{RNA Isolation}

Isolation of high-purity RNA is an important step for a successful RTPCR. When high sample throughput is required, the isolation procedure should be quick and reliable. The QIAamp RNA Blood Mini Kit and the High Pure $^{\mathrm{TM}}$ RNA Isolation Kit (Roche Applied Science) were compared for the quality and yield of isolated total RNA.

\section{Reproducibility of the cDNA Synthesis}

We obtained reproducible results with the QIAamp kit both in spectrophotometer measurements of total RNA concentration and in PCR quantification. When starting from one multiple sclerosis patient blood sample aliquoted into 10 independent total
RNA isolations, the CV for the photometric measurement $\left(\mathrm{A}_{260}\right)$ was $6.7 \%$. When amplifying the 10 resulting cDNA aliquots from each of the RNA isolations individually and quantifying the absolute copy numbers with the appropriate imported standard curve, the $\mathrm{CV}$ was $25.5 \%$ (copy number $\overline{\mathrm{x}} \pm \mathrm{SD}$ : $3116 \pm 794 ; \mathrm{cV}$ of $\mathrm{Cp}=1.3 \%$ ) for PBGD, $21 \%$ for IFN- $\gamma(5954 \pm 1249$; $\mathrm{CV}$ of $\mathrm{Cp}=1.1 \%$ ), and $18 \%$ for IL-4R $(20096 \pm 3647 ; \mathrm{cv}$ of $\mathrm{Cp}=1.1 \%)$.

\section{Determination of the Linearity of cDNA Synthesis}

Because of the individual physiological status of each multiple sclerosis patient, the amount of total RNA isolated varied considerably (range of $0.2-1.2$ $\mu \mathrm{g} / 9.5 \mu \mathrm{L}$ ). When quantifying low RNA contents, photometric measurements at $\mathrm{A}_{260}$ were not reliable enough to allow accurate fixed amounts of total RNA to be added to the cDNA synthesis reactions. Therefore, a constant volume $(9.5 \mu \mathrm{L})$ of total RNA was used instead. To ensure that varying amounts of RNA did not affect the reverse transcription, the range of linearity of the cDNA synthesis as a function of vary-

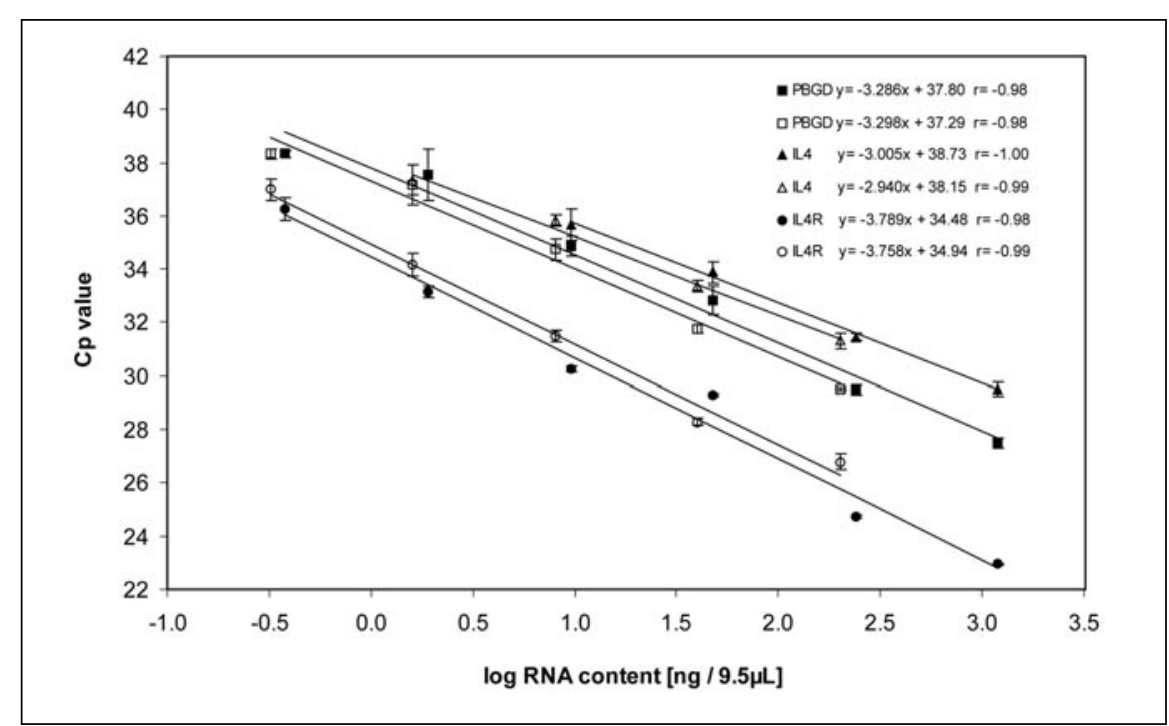

Figure 1. Linear relationship of cDNA synthesis as a function of RNA content and gene expression strength. Undiluted and serially 1:5 dilutions (MS2 RNA: $10 \mathrm{ng} / \mu \mathrm{L}$ ) of two differently concentrated RNA extracts from MS patients [1.2 $\mu \mathrm{g} / 9.5 \mu \mathrm{L}$ (black symbols) or $0.2 \mu \mathrm{g} / 9.5 \mu \mathrm{L}$ (open symbols)] were used for cDNA synthesis and subsequent PCR amplification (45 cycles) in quintuple for PBGD (squares) as a housekeeping gene, IL-4 (triangles) as a parameter with gene expression strength less than that of PBGD, and IL-4R (circles) as a parameter with gene expression strength greater than that of PBGD. The curves were obtained by plotting the mean values $(n=5)$ of $\log$ RNA content $(\mathrm{ng} / 9.5 \mu \mathrm{L})$ versus the mean $(n=5)$ of the $\mathrm{Cp}$ values and were given in the form $\mathrm{y}=\mathrm{mx}+\mathrm{b}$. The error bars show the standard deviation. The regression coefficient was greater than or equal to 0.98 in all cases. 
ing RNA contents and varying gene expression strength of the different parameters measured was analyzed. Therefore, two total RNA isolations $(1.2 \mu \mathrm{g} / 9.5 \mu \mathrm{L}$ or $0.2 \mu \mathrm{g} / 9.5 \mu \mathrm{L})$ were serially diluted five times in 1:5 steps with MS2 RNA (final concentration, 10 $n g / \mu \mathrm{L})$. Undiluted and diluted RNAs were used for cDNA synthesis, and all were amplified in PCR in quintuple for the five different parameters. In Figure 1 , results were shown for PBGD as the housekeeping gene used for the relative quantification, for IL-4 as a parameter with a gene expression strength less than that of PBGD, and for IL-4R as a parameter with a gene expression strength greater than that of PBGD. For all three parameters, a linear relationship was observed over at least three orders of magnitude, irrespective of the RNA content added to the reverse transcription reaction and of the parameterdependent gene expression strength. The linearity regarding the RNA content applied over a range from 1200 to $8 \mathrm{ng} / 9.5 \mu \mathrm{L}$ for IL-4 and from 1200 to $0.3 \mathrm{ng} / 9.5 \mu \mathrm{L}$ for PBGD and IL-4R. The limit of detection of the total RNA concentration was approximately 0.8 $\mu \mathrm{g} / \mathrm{mL}$ for IL-4 and approximately 0.03 $\mu \mathrm{g} / \mathrm{mL}$ for PBGD and IL-4R. An influence of the varying RNA contents from our multiple sclerosis patients on mRNA quantification can be excluded within the minimum range of 1.2 $0.008 \mu \mathrm{g}$ (IL-4).

\section{Reproducibility of the Real-Time PCR: Intra- and Inter-Assay Variation}

Reproducibility of the real-time PCR was examined by intra- and inter-assay amplification. The intra-assay precision was determined by 15 repeated measurements of a patient cDNA $(0.81 \mu \mathrm{g}$ RNA/9.5 $\mu \mathrm{L}$ ) within one LightCycler run. The amplification and quantification was done for PBGD and IFN- $\gamma$ using the appropriate imported standard curve. The $\mathrm{CV}$ for the absolute copy numbers was $11.2 \%$ for PBGD (copy number $\overline{\mathrm{x}} \pm \mathrm{SD}: 1900 \pm 212$; $\mathrm{CV}$ of $\mathrm{Cp}=$ $1.1 \%)$, and $13.5 \%$ for IFN- $\gamma(265 \pm 36$; $\mathrm{CV}$ of $\mathrm{Cp}=0.6 \%$ ). Inter-assay variation was investigated in 10 different runs performed on the same day for the amplification of IFN- $\gamma$ with the same pa- tient cDNA and with different dilutions $\left(1 \times 10^{5}, 1 \times 10^{4}\right.$, and $\left.1 \times 10^{2}\right)$ of the IFN- $\gamma$ plasmid standard. The quantification of cDNA and standard plasmid was done using the imported IFN- $\gamma$ standard curve. The $\mathrm{CV}$ for the absolute copy numbers of the patient cDNA was $20.4 \%$ (copy number $\bar{x} \pm$ SD: $368 \pm 75$; $\mathrm{CV}$ of $\mathrm{Cp}=0.8 \%)$. Those of the plasmid standard dilutions were $8.7 \%$ for $1 \times$ $10^{5}(153390 \pm 13374 ; \mathrm{cV}$ of $\mathrm{Cp}=$ $0.9 \%), 13.7 \%$ for $1 \times 10^{4}(12811 \pm$ 1759 ; $\mathrm{CV}$ of $\mathrm{Cp}=1.0 \%$ ), and $15.3 \%$ for $1 \times 10^{2}(86 \pm 13 ; \mathrm{cV}$ of $\mathrm{Cp}=0.8 \%)$.

\section{Construction of External Plasmid Standard Curves}

The appropriate homologous DNA plasmid standards, which possessed the same binding sides for primers and hybridization probes as did the target, were used for quantification of cytokine targets and PBGD. To achieve a reliable standard curve for each measured parameter a separate standard curve was constructed by serially (1:10) diluting the plasmids in MS2 RNA over the appropriate concentration range. Every dilution point was PCR-amplified in five replicates. To check for reproducibility, independent standard curves were constructed on different days. Figure 2 shows the standard curves for PBGD, IL-4, IL-4R and IFN- $\beta$. The standard curve for IFN- $\gamma$ (y $=-3.305 \mathrm{x}+38.92$, Error $=0.0546, \mathrm{r}=$ -1.00 ) is not shown because it is congruent to that of PBGD. A linear relationship was detected over five orders of magnitude for all parameters, when the means of the log values of the LightCycler-calculated copy numbers were plotted versus the means of the $\mathrm{Cp}$ values. The error bars represent the $\mathrm{x}$ axis and $y$-axis standard deviations of both measured values for the 5-fold measurements. The standard deviations for the log of the calculated copy numbers were between \pm 0.029 and \pm 0.291 . The standard deviations for the $\mathrm{Cp}$ values were between \pm 0.094 and \pm 0.960 . Since there is a $50 \%$ probability of amplifying one copy only, 2-3 of 5 reactions should show an amplification signal (IL- $4, n=3$; IFN- $\gamma, n=2$; IFN- $\beta, n$ $=1$ ). This number of reactions for the $10^{0}$ dilution was reproducible when independent standard curves were measured. As expected, the standard deviation increased with decreasing copy number. The greatest deviation was for the $10^{0}$ dilution and in particular for IFN- $\gamma( \pm 0.291)$. Previous measure-

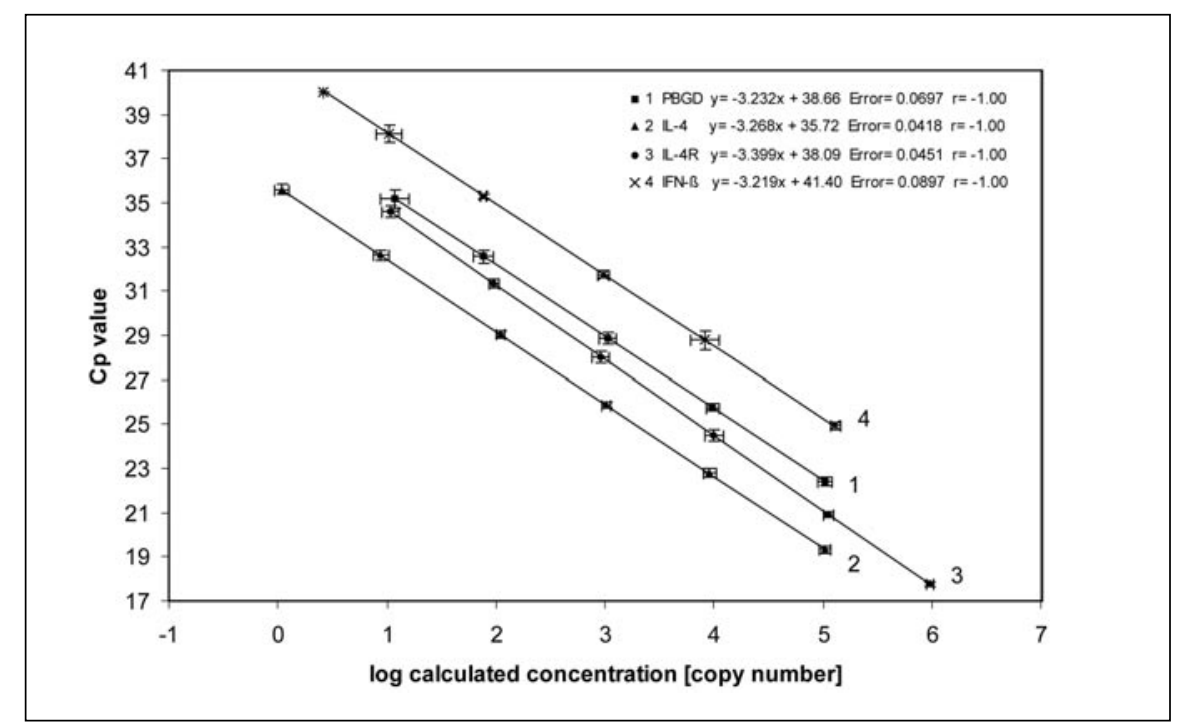

Figure 2. Constructed plasmid standard curves for the quantification of multiple sclerosis patient cDNA. 1:10 dilutions (MS2 RNA: $10 \mathrm{ng} / \mu \mathrm{L}$ ) of DNA plasmid standards (log range depends on the target) were used for PCR amplification in quintuple for 45 cycles (IFN- $\beta$, 50 cycles) of PBGD (square), IL-4 (triangle), IL-4R (circle), and IFN- $\beta$ (cross). The curves were obtained by plotting the mean values of logcalculated concentration (copy number) versus the $\mathrm{Cp}$. The error bars show the standard deviation $(n=5$, except for the $10^{0}$ dilution points). The amplification efficiencies differed between 1.97-2.02 (IFN- $\gamma, \mathrm{y}=$ $-3.305 x+38.92$, Error $=0.0546, r=-1.00$ ). The error values of the standard curves are the mean squared errors calculated by the LightCycler software. The regression coefficient was -1.00 in all cases. 
Table 2. Reproducibility of Imported Standard Curves for PBGD, IL-4, and IL-4R

\begin{tabular}{|c|c|c|c|c|c|c|}
\hline Parameter & Run & Cp STD $10^{3} 1$. & Cp STD $10^{3} 2$. & Cp $10^{3}$ "Unknown" & Slope & Y-Intercept \\
\hline \multirow{10}{*}{ PBGD } & 1 & 29.99 & 29.77 & 29.86 & -3.232 & 39.58 \\
\hline & 2 & 29.56 & 28.76 & 29.67 & -3.232 & 38.86 \\
\hline & 3 & 28.83 & 28.86 & 29.01 & -3.232 & 38.55 \\
\hline & 4 & 29.34 & 29.14 & 28.90 & -3.232 & 38.94 \\
\hline & 5 & 29.43 & 29.25 & 29.42 & -3.232 & 39.04 \\
\hline & 6 & 29.44 & 29.49 & 28.95 & -3.232 & 39.16 \\
\hline & 7 & 29.05 & 29.11 & 29.34 & -3.232 & 38.78 \\
\hline & 8 & 29.21 & 28.74 & 29.59 & -3.232 & 38.67 \\
\hline & 9 & 28.55 & 28.14 & 28.67 & -3.232 & 38.04 \\
\hline & 10 & 28.65 & 28.43 & 28.12 & -3.232 & 38.24 \\
\hline CV (\%) & & 1.44 & 1.59 & 1.71 & 0.00 & 1.09 \\
\hline \multirow[t]{10}{*}{ IL-4 } & 1 & 25.90 & 26.13 & 26.28 & -3.268 & 35.82 \\
\hline & 2 & 25.62 & 25.46 & 25.82 & -3.268 & 35.35 \\
\hline & 3 & 25.64 & 25.45 & 25.45 & -3.268 & 35.35 \\
\hline & 4 & 25.93 & 25.61 & 25.51 & -3.268 & 35.57 \\
\hline & 5 & 25.80 & 25.71 & 25.85 & -3.268 & 35.56 \\
\hline & 6 & 26.12 & 26.44 & 26.18 & -3.268 & 35.97 \\
\hline & 7 & 26.62 & 26.54 & 26.09 & -3.268 & 36.38 \\
\hline & 8 & 25.99 & 26.01 & 26.45 & -3.268 & 35.80 \\
\hline & 9 & 25.83 & 25.97 & 25.86 & -3.268 & 35.70 \\
\hline & 10 & 26.09 & 26.31 & 26.15 & -3.268 & 36.00 \\
\hline CV (\%) & & 1.05 & 1.45 & 1.19 & 0.00 & 0.84 \\
\hline \multirow[t]{10}{*}{ IL-4R } & 1 & 27.27 & 27.84 & 27.51 & -3.399 & 37.75 \\
\hline & 2 & 27.89 & 27.87 & 27.53 & -3.399 & 38.08 \\
\hline & 3 & 27.01 & 27.11 & 27.45 & -3.399 & 37.26 \\
\hline & 4 & 28.10 & 27.55 & 27.64 & -3.399 & 38.03 \\
\hline & 5 & 27.01 & 27.93 & 27.79 & -3.399 & 37.67 \\
\hline & 6 & 26.46 & 26.66 & 27.15 & -3.399 & 36.76 \\
\hline & 7 & 26.80 & 26.70 & 26.84 & -3.399 & 36.95 \\
\hline & 8 & 26.59 & 27.14 & 27.03 & -3.399 & 37.07 \\
\hline & 9 & 26.83 & 26.82 & 27.01 & -3.399 & 37.02 \\
\hline & 10 & 26.64 & 26.69 & 26.56 & -3.399 & 36.86 \\
\hline CV (\%) & & 1.92 & 1.82 & 1.37 & 0.00 & 1.26 \\
\hline
\end{tabular}

ments of multiple sclerosis patient cDNA showed that for the quantification of IFN- $\gamma$ a standard concentration range down to $10^{1}$ is sufficient. When quantification is required in a range from $10^{1}$ to $10^{0}$, at least duplicates of the samples should be measured.

\section{Importing External Standard Curves to Different PCR Runs}

The LightCycler software version 3.5 provides a tool to import the con- structed standard curves to different PCR runs. Existing standard curve data were used to create separate files (*.xsc) that could be imported into other runs for quantification of cDNA targets. A prerequisite for importing an external standard curve is that a sample with known concentration has to be included in every run. For this we used duplicate samples of the appropriate aliquoted $10^{3}$ standard dilution point (see Construction of the External Plasmid Standard Curves section), which we have designated as a standard, and also one $10^{3}$ standard dilution point, which we have designated as an unknown sample (further called: $10^{3}$ triplicate). When importing a standard curve to a run without standard curve data, the slope will remain constant and correspond to that of the appropriate parameter-dependent standard curve. However, the imported standard curve will be shifted along the $\mathrm{y}$-axis $(\mathrm{Cp})$ depending on the $\mathrm{Cp}$ values of measured $10^{3}$ duplicate standards. A change of this $\mathrm{Cp}$ value 
would lead to a change of all other $\mathrm{Cp}$ values in the standard curve; therefore, the absolute concentration (copy numbers) of standards and unknowns would change. When importing standard curves into different runs with unknown samples, it is very important that the $\mathrm{Cp}$ values of the $10^{3}$ triplicate (standards and unknown) remain constant and correspond to the $\mathrm{Cp}$ value of the $10^{3} \mathrm{stan}-$ dard of the appropriate imported standard curve. The $10^{3}$ standard dilutions of the different parameters can be used for at least six months, when diluted in MS2 RNA (final concentration, 10 $\mathrm{ng} / \mu \mathrm{L}$ ), aliquoted in siliconized tubes, and stored at $-20^{\circ} \mathrm{C}$. With the use of the aliquoted and stored $10^{3}$ dilutions, it was easy practicable, and we could achieve reproducible standard curves. Otherwise, we did not use the run for quantification.

\section{Reproducibility of the Imported Plasmid Standard Curves}

To check for variations of the $10^{3}$ triplicate (standards and unknown), 10 separate runs with imported standard curves for PBGD, IL-4, and IL-4R (Table 2) were compared with 10 repeated runs of an entire IFN- $\gamma$ standard curve with a concentration range from $10^{5}$ to $10^{2}$. The IFN- $\gamma$ standard curves were constructed on the same day with a single PCR amplification for all dilution points. The $10^{3}$ triplicate was measured on different days with independent aliquots. The inter-assay coefficients of variation of the $C p$ values of the measured $10^{3}$ triplicate were between $1 \%$ and $2 \%$ for the imported standard curves of the three parameters. As mentioned already, the slopes of the imported standard curves remained constant, and the calculated amplification efficiencies were 2.04 for PBGD, 2.02 for IL-4, and 1.97 for IL-4R. The coefficient of variation of the y-intercept varied between $0.8 \%$ and $1.3 \%$ and was therefore comparable to that of the IFN$\gamma$ standard curves $(0.8 \%)$. As long as the $\mathrm{Cp}$ values of the $10^{3}$ triplicate remained constant, it was possible to use imported standard curves for quantification of unknowns measured in different runs.

\section{PBGD as Reference for the Relative Quantification of Cytokine mRNAs}

The endogenous gene expression of the human PBGD housekeeping gene was used to normalize the gene expression of different cytokine targets. It serves as a reference to correct for differences in the amount of total RNA added to a reaction and to compensate for variations in target recovery during nucleic acid extractions and the presence of inhibitors. PBGD is expressed in a broad range of tissues in a relatively stable expression level and without pseudogenes (4). It belongs to the lowabundance class of mRNAs (8). This makes PBGD a suitable reference, especially for the quantification of cytokines. Although PBGD expression levels are comparable in various tissues, they may vary under therapeutic treatment conditions. Therefore, we have compared untreated $(n=30)$ and $\beta$-interferon-treated $(n=57)$ multiple sclerosis patients for the expression levels of PBGD. Patient samples were measured for both groups over a period of 12 months at three-month intervals. On the basis of the intra- and inter-individual variation of the absolute $\mathrm{PBGD}$ values in both groups, we have compared the mean values of the differences of the successive three-month measurements versus a baseline value for the untreated and treated patients, respectively. The data were analyzed using the Mann-Whitney U-test for unrelated values. An influence of the $\beta$-in-
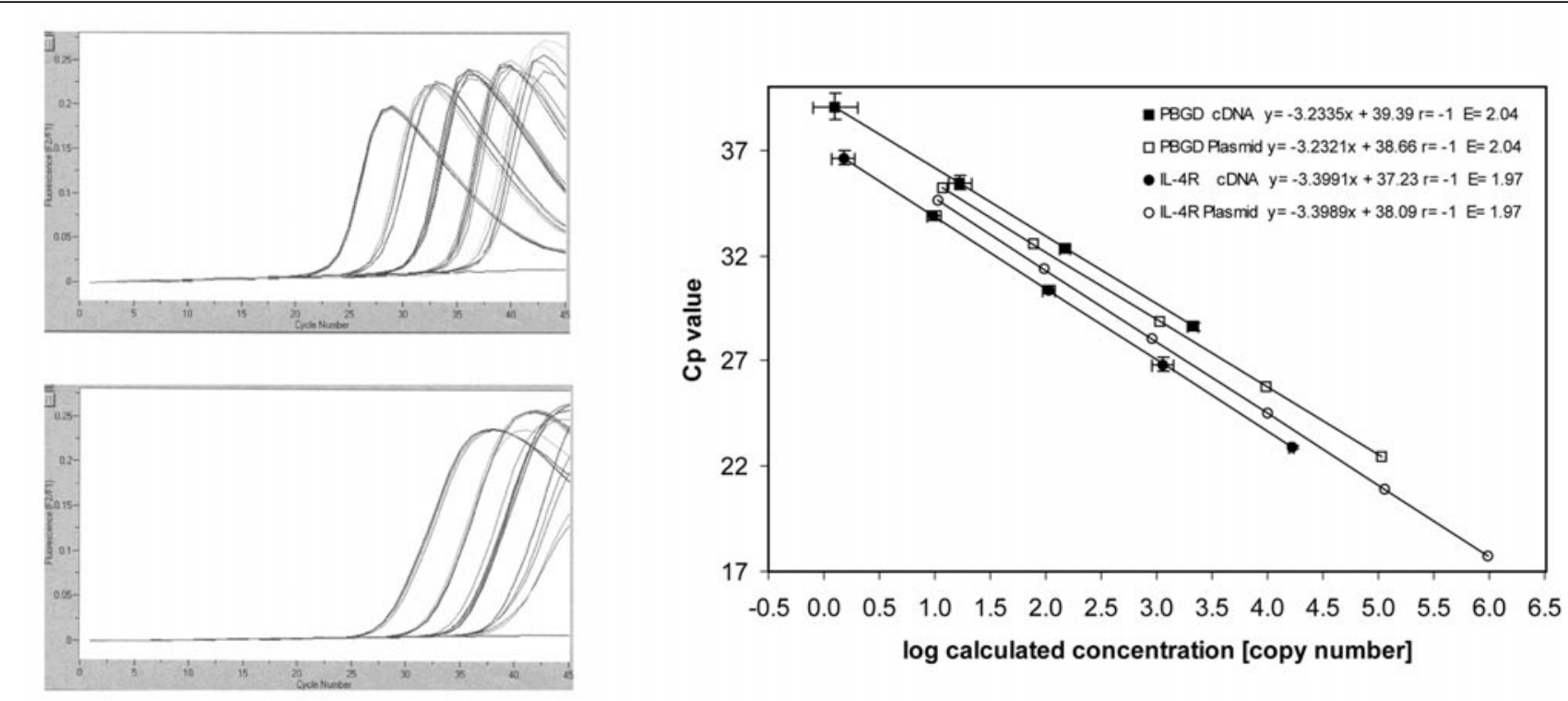

Figure 3. PCR amplification efficiencies of patient cDNA compared to plasmid standard. Undiluted (1:1) and 4-fold serial 1:10 dilutions (MS2 RNA, 10 $\mathrm{ng} / \mu \mathrm{L}$ ) of a patient cDNA were PCR-amplified for PBGD and IL-4R. Left side: PCR amplification curves for the limiting dilution series of the cDNA. Right side: cDNA standard curves (PBGD, black square; IL-4R, black circle) compared to plasmid standard curves (PBGD, open square; IL-4R, open circle) when plotting the mean of the log values of the calculated copy numbers versus the mean of the $\mathrm{Cp}$ values. The error bars show the standard deviation $(1: 1, n=3$; 1:10-1:10000, $n=5$ ) of the cDNA measurements (for plasmid standard curves, see Figure 2). 
terferon therapy on the PBGD levels could not been found ( $P=0.608$; difference $\overline{\mathrm{x}} \pm$ SD: untreated: $359.06 \pm$ 1220.25; treated: $514.37 \pm 1317.37$ ).

\section{PCR Amplification Efficiency of Plasmid Standard Compared to Patient cDNA}

Theoretically, the use of external homologous DNA plasmid standards for the quantification of each different parameter ensures identical amplification efficiencies of standard and cDNA. In practice, there are differences in efficiencies due to inhibitors and the different composition of highly pure plasmid solution compared to the pool of cDNAs. To check the extent of these differences, undiluted and serial 1:10 dilutions of MS2 RNA-diluted patient cDNA were measured for every parameter. The several dilutions of the cDNA were PCR-amplified in quintuple, the undiluted cDNA in triplicate. The absolute copy numbers of the different parameters were quantified with the appropriate imported plasmid standard curve. Figure 3 shows exemplary data for PBGD and IL-4R. On the left side, it shows the amplification curves as displayed by the LightCycler software for the limiting dilution series of the patient cDNA. The PCR limit of detection was 1.4 copies ( $\overline{\mathrm{x}}$ of $n=5 ; \mathrm{SD}=$ \pm 0.5 ) for PBGD and 1.5 copies ( $\overline{\mathrm{x}}$ of $n$ $=5 ; \mathrm{SD}= \pm 0.4$ ) for IL-4R. On the right side, it shows the cDNA standard curves when plotting the means of the log values of the LightCycler-calculated copy numbers versus the means of the $\mathrm{Cp}$ values. Both cDNA curves showed the same slope compared to the appropriate plasmid standard curve, demonstrating identical amplification efficiency for target samples and plasmid standard.

Here we have developed a two-step RT-PCR system using hybridization probes and external plasmid standards for the relative quantification of cytokines and their receptors. This system has high sensitivity and reproducibility and is linear over a wide concentration range for both cDNA synthesis and PCR amplification. The opportunity to use imported standard curves of the external plasmid standards for the determination of absolute copy numbers allows precise and fast measurements, which can com- pensate for the relatively extensive expenditure for method optimization and standard curve construction.

\section{ACKNOWLEDGMENTS}

This work was supported by Roche Applied Science. Special thanks to Dr. Stephan Stubner and Katja Meuser (MPI for terrestrial microbiology, Marburg, Germany) for their technical help and to Dr. Peter Dunfield (MPI for terrestrial microbiology) for reading the manuscript.

\section{REFERENCES}

1.Blaschke, V., K. Reich, S. Blaschke, S. Zipprich, and C. Neumann. 2000. Rapid quantitation of proinfammatory and chemoattractant cytokine expression in small tissue samples and monocyte-derived dendritic cells: validation of a new real-time RT-PCR technology. J. Immunol. Methods 246:79-90.

2.Bustin, S.A. 2000. Absolute quantification of mRNA using real-time reverse transcription polymerase chain reaction assays. J. Mol. Endocrinol. 25:169-193.

3.Clegg, R.M. 1995. Fluorescence resonance energy transfer. Curr. Opin. Biotechnol. 6:103-110

4.Finke, J., R. Fritzen, P. Ternes, W. Lange, and G. Dölken. 1993. An improved strategy and a useful housekeeping gene for RNA analysis from formalin-fixed, paraffin-embedded tissues by PCR. BioTechniques 14:448453.

5.Freeman, W.M., S.J. Walker, and K.E. Vrana. 1999. Quantitative RT-PCR: pitfalls and potential. BioTechniques 26:112-125.

6.Higuchi, R., C. Fockler, G. Dollinger, and R. Watson. 1993. Kinetic PCR analysis: realtime monitoring of DNA amplification reactions. BioTechnology 11:1026-1030.

7.Kühne, B.S. and P. Oschmann. 2001. Quantification of cytokines with "real-time" RTPCR and ELISA to find a surrogate marker for therapy-control in multiple sclerosis patients. Multiple Sclerosis 7(suppl 1):S35.

8.Mensink, E., A. van de Locht, A. Schattenberg, E. Linders, N. Schaap, A. Geurts van Kessel, and T. de Witte. 1998. Quantitation of minimal residual disease in Philadelphia chromosome positive chronic myeloid leukaemia patients using real-time quantitative RT-PCR. Br. J. Haematol. 102:768-774.

9.Stordeur, P., L.F. Poulin, L. Craciun, L. Zhou, L. Schandené, A. de Lavareille, S. Goriely, and M. Goldman. 2002. Cytokine mRNA quantification by real-time PCR. J. Immunol. Methods 259:55-64.

10.Wittwer, C.T., M.G. Herrmann, A.A. Moss, and R.P. Rasmussen. 1997. Continuous fluorescence monitoring of rapid cycle DNA amplification. BioTechniques 22:130-138.

11.Wittwer, C.T., K.M. Ririe, R.V. Andrew,
D.A. David, R.A. Gundry, and U.J. Balis. 1997. The LightCycler ${ }^{\mathrm{TM}}$ : a microvolume multisample fluorimeter with rapid temperature control. BioTechniques 22:176-181.

Received 27 March 2002; accepted 3 July 2002.

Address correspondence to:
Dr. Benedikte Sabina Kühne
Neurologische Universitätsklinik
Neurochemisches Labor
Am Steg 16
D-35392 Gießen
Germany
e-mail: sabina.kuehne@neuro.med.uni-
giessen.de

For reprints of this or any other article, contact Reprints@BioTechniques.com 